\title{
Precision tests of the Standard Model at the LHC with the ATLAS and CMS detectors
}

\author{
Gabriella Pásztor ${ }^{* \dagger}$ \\ MTA-ELTE Lendület CMS Particle and Nuclear Physics Group, Eötvös Loránd University, \\ Budapest \\ E-mail: Gabriella.Pasztor@cern.ch
}

\section{on behalf of the ATLAS and CMS Collaborations}

Precision measurements provide a critical test of the consistency and limits of validity of the Standard Model (SM) and could point toward New Physics and constrain the parameters of the extensions of the SM. The ultimate aim is to measure directly the electroweak mixing angle $\left(\theta_{\mathrm{W}}\right)$ and the mass of the $W$ boson $\left(m_{W}\right)$ with a precision that is competitive with the global fit uncertainties. To achieve an excellent understanding of experimental and parton distribution function uncertainties affecting the determination of $\sin ^{2} \theta_{\mathrm{W}}$ and $m_{W}$, a rich program of $Z$ and $W$ boson studies are performed, including the measurements of differential production cross-sections as a function of various kinematic variables (like the transverse boson momenta, rapidity and additional jet variables), the $Z \rightarrow \ell^{+} \ell^{-}$angular coefficients and forward - backward asymmetry as well as the $W$ boson production charge asymmetry. Recent results using the LHC Run 1 and Run 2 data are summarised in this article from the ATLAS and CMS collaborations.

International Conference on Precision Physics and Fundamental Physical Constants - FFK2019

9-14 June, 2019

Tihany, Hungary

\footnotetext{
* Speaker.

$\dagger$ The author wishes to thank for their support the Hungarian Academy of Sciences "Lendület" (Momentum) Program (LP 2015-7/2015) and the National Research, Development and Innovation Office of Hungary (NKFIA K 124845 and $\mathrm{K} 128713)$.
} 


\section{Introduction}

Precise electroweak measurements coupled with precise theoretical calculations successfully predicted the masses of the $W$ and $Z$ bosons, the top quark and most recently of the Higgs boson even before their direct observation. Such measurements are essential to test the consistency of the Standard Model (SM), explore its limits of validity, and they could also point toward New Physics and constrain the parameters of beyond the SM theories.

The electroweak (EW) gauge and Higgs sector is described by four parameters, which are often chosen as the fine structure constant $\alpha$, the Fermi coupling constant $\left(G_{\mathrm{F}}\right)$ and the masses of the $Z$ and Higgs bosons $\left(m_{Z}\right.$ and $\left.m_{H}\right)$. Other parameters, such as the electroweak mixing angle $\left(\theta_{\mathrm{W}}\right)$, the mass of the $W$ boson $\left(m_{W}\right)$, the gauge couplings and the parameters of the Higgs potential are then predicted by theory. By performing measurements of the chosen and derived parameters, the model becomes overconstrained and global fits can verify its consistency.

The ultimate aim of the LHC electroweak precision physics programme (not discussing here the Higgs sector that is summarised in Ref. [1]) is to measure directly the EW mixing angle to 0.0001 and the $W$ boson mass to $10 \mathrm{MeV}$, i.e. with precisions that are competitive with the global fit uncertainties: $\sin ^{2} \theta_{\mathrm{W}}=0.23153 \pm 0.00006$ and $m_{W}=80.354 \pm 0.007 \mathrm{GeV}$ [5], shown in Fig. 1 (top). The current world averages are about a factor two less precise: $\sin ^{2} \theta_{\mathrm{W}}=0.23152 \pm 0.00016$ and $m_{W}=80.379 \pm 0.013 \mathrm{GeV}[2]$.

To achieve this goal an excellent understanding of uncertainties originating from experimental sources and parton distribution functions (PDFs) are needed. The ATLAS [3] and CMS [4] experiments thus pursue a rich program of $Z$ and $W$ boson studies. In the following a summary of recent results in this area is given, including the measurements of differential production cross-sections as a function of various kinematic variables (like the transverse boson momenta, rapidity and additional jet variables), the $Z \rightarrow \ell^{+} \ell^{-}$angular coefficients and forward - backward asymmetry as well as the $W$ boson production charge asymmetry. These results are based on the LHC Run 1 and Run 2 data collected at center-of-mass energies of $\sqrt{s}=7,8$ and $13 \mathrm{TeV}$ with an integrated luminosity of about $5 \mathrm{fb}^{-1}, 20 \mathrm{fb}^{-1}$ and 3-80 $\mathrm{fb}^{-1}$, respectively. As these measurements typically require the best detector calibration possible, the full Run 2 dataset collected at $\sqrt{s}=13 \mathrm{TeV}$ between 2015 and 2018 amounting to approximately $150 \mathrm{fb}^{-1}$ per experiment with an average number of $\mathrm{p}-\mathrm{p}$ interactions per bunch crossing of $\langle\mu\rangle \approx 34$ is still being analysed. A summary of the available cross-section measurements of various SM processes from ATLAS is shown in Fig. 2, compared to theoretical predictions calculated at least at next-to-leading order (NLO) in perturbative quantum chromodynamics (pQCD). It shows an impressive agreement over 14 orders of magnitude in cross-section from the most abundant to the rarest processes observed so far, attesting the triumph of both experimental and theoretical efforts.

\section{Top quark mass}

The top quark mass plays a very important role in testing the SM self-consistency as it gives significant contribution to the values of EW parameters via quantum corrections (see Fig. 1 (bottom right)). Its value is also critical for the stability of the EW vacuum. Direct measurements use the decay products to reconstruct the top quark mass. After comparing the data to Monte Carlo (MC) 

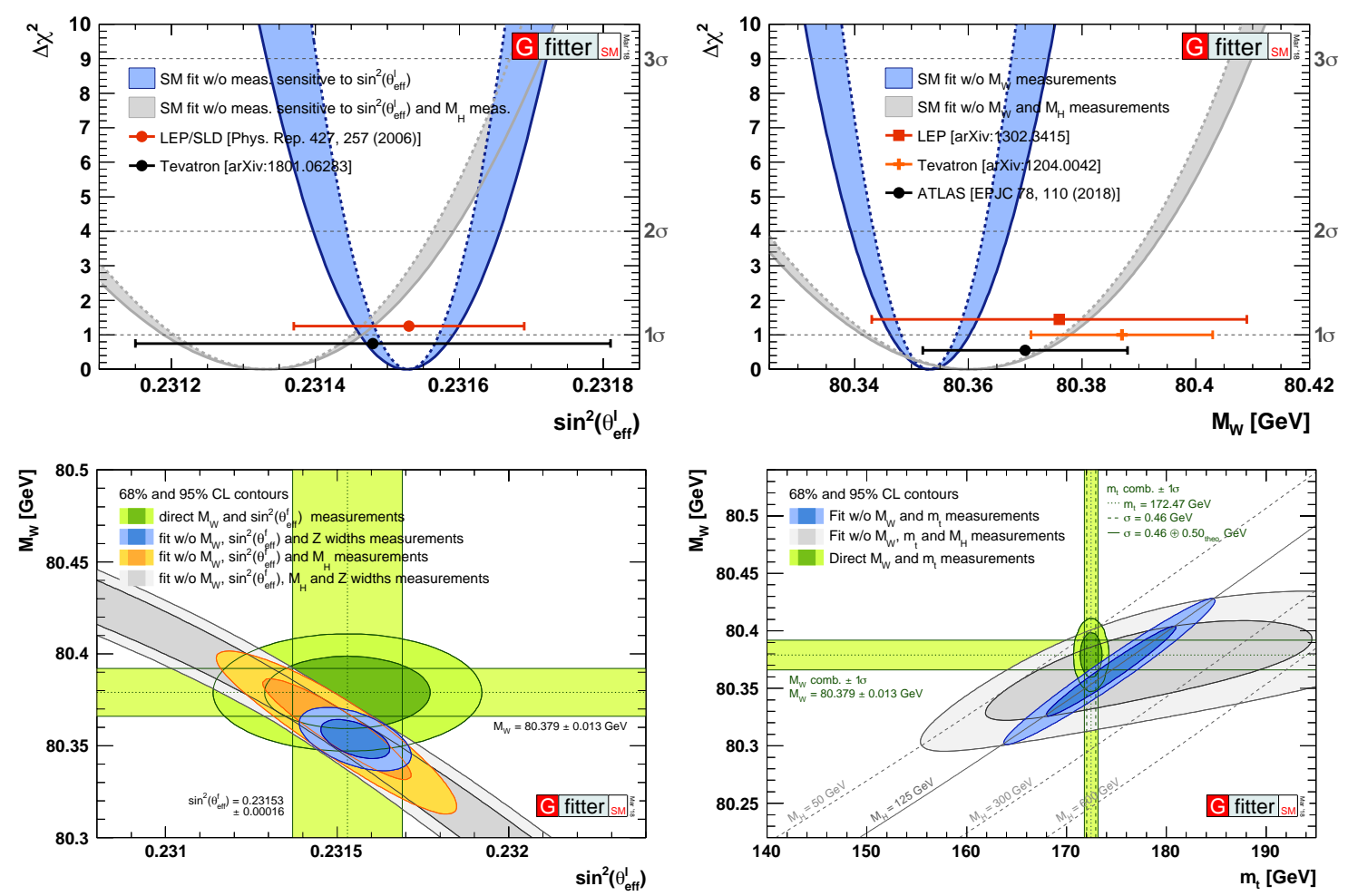

Figure 1: (top) $\Delta \chi^{2}$ as a function of (left) the effective weak mixing angle and (right) the $W$ boson mass in the global SM fit by the Gfitter group (blue band). The result of the fit without the Higgs boson mass measurement is also shown (grey band). All precision observables sensitive to $\sin ^{2} \theta_{\text {eff }}$ on the left and direct measurements of $m_{W}$ on the right are excluded from the fit. The direct measurement results are indicated by dots with $1 \sigma$ error bars. (bottom) Contours of $68 \%$ and $95 \%$ confidence level obtained from scans of fits with fixed variable pairs of (left) $m_{W}$ vs. $\sin ^{2} \theta_{\text {eff }}$ and (right) $m_{W}$ vs. $m_{t}$. The narrower blue and larger grey allowed regions are the results of the fit including and excluding the $m_{H}$ measurement, respectively. The horizontal bands indicate the $1 \sigma$ regions of the direct measurements. From Ref. [5].

simulation predictions, the measured value is interpreted as the pole mass. Indirect measurements on the other hand determine either the pole mass or the running mass in the minimal subtraction (MS) scheme from the cross-section or from differential distributions. As illustrated in Fig. 3, the various measurements from ATLAS [7] and CMS [8] agree well with each other within the experimental uncertainty of about $0.3 \%$ and indicate a somewhat smaller value than that of the Tevatron combination, moving the world average to $172.9 \pm 0.4 \mathrm{GeV}[2]$.

\section{3. $W$ boson mass}

The first LHC measurement of the $W$ boson mass was published by ATLAS based on its $\sqrt{s}=7 \mathrm{TeV}$ data of $W \rightarrow e v$ and $W \rightarrow \mu v$ decays [9]. The $W$ mass is derived from a template fit to the lepton transverse momentum $\left(p_{\mathrm{T}}\right)$ distribution (shown in Fig. 4 (left)) or to the transverse mass $\left(m_{\mathrm{T}}\right)$ calculated from the lepton four-momenta and the missing transverse momentum. While the first is sensitive to the theoretical modelling of the $W$ boson transverse momentum distribution, the latter depends on the hadronic recoil momentum calibration. Templates are taken from a POWHEG 


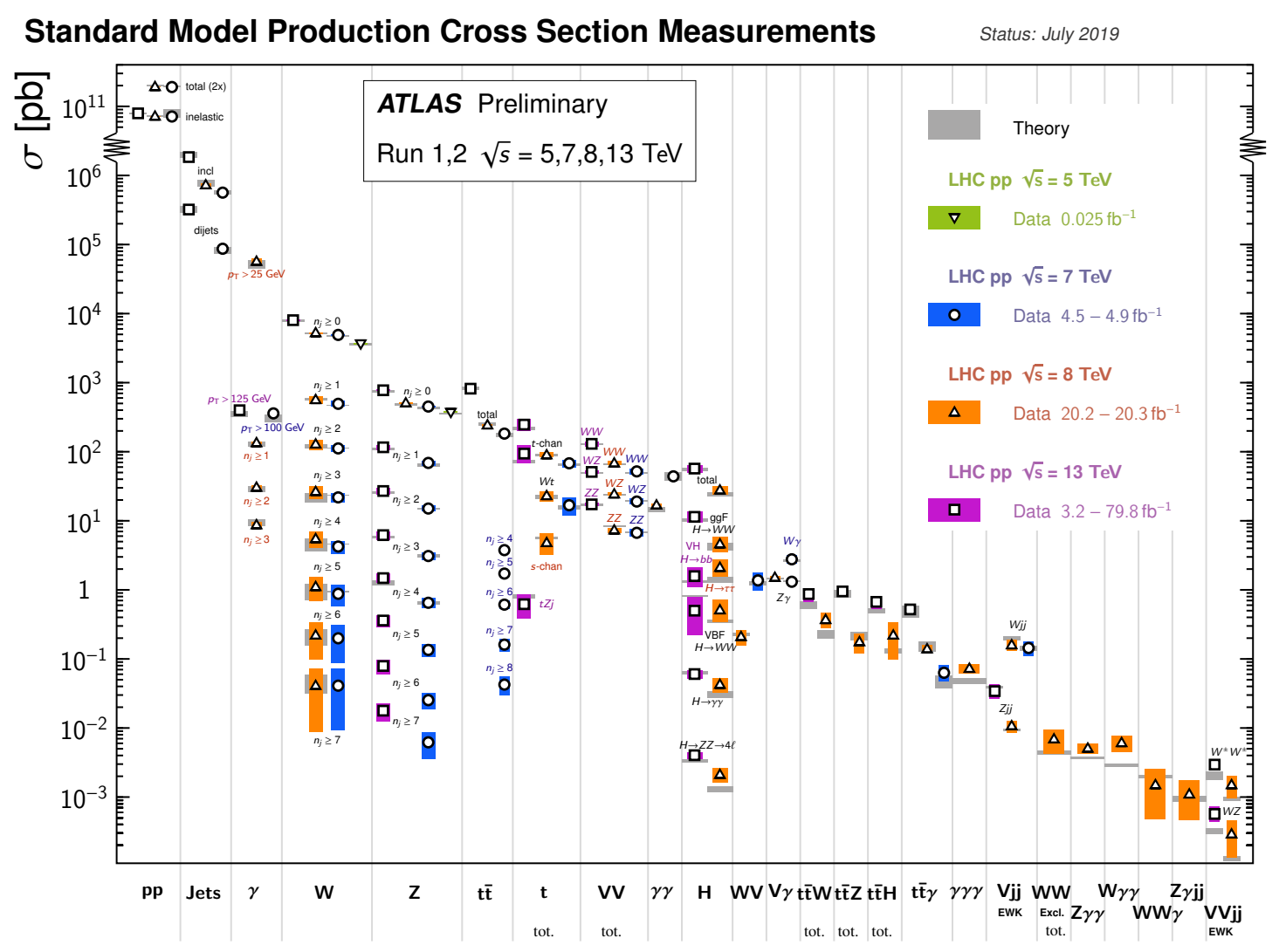

Figure 2: Summary of Standard Model total and fiducial production cross-section measurements by ATLAS, corrected for branching fractions, compared to the higher order theoretical expectations [6].

+ PYTHIA8 simulation and then reweighted to the best theoretical model. DYNNLO is used to model the rapidity distribution and $W$ polarisation, while the transverse momentum distribution is taken from PYTHIA8 using the AZ tune [10]. $Z \rightarrow e^{+} e^{-}, \mu^{+} \mu^{-}$events are heavily used for modelling validation as well as detector calibration.

The main experimental challenges come from the electron, muon and hadron recoil momentum scale calibration, contributing $6.4 \%, 6.6 \%$ and $2.9 \%$ uncertainties, comparable to the statistical error of $6.8 \%$. The multijet background is measured using template fits in regions with modified kinematic selection with the template shapes acquired using inverted lepton isolation requirements. The background uncertainty amounts to $4.5 \%$. The dominant uncertainty comes from physics modelling. Parton distribution function uncertainties are the largest (9.2\%). QCD related uncertainties (8.3\%) affect the $W$ transverse momentum distribution as well as extrapolations from $Z$ measurements to $W$ final states. Electroweak uncertainties are subdominant $(5.5 \%)$. The total uncertainty of the final combined measurement is thus $18.5 \%$. The individual measurements are compatible with each other as illustrated in Fig. 4 (middle), the $\chi^{2}$ per degrees of freedom is $29 / 27$. The result $m_{W}=80370 \pm 7$ (stat.) \pm 11 (exp. syst.) \pm 14 (mod. syst.) $\mathrm{MeV}=80370 \pm 19 \mathrm{MeV}$ is close in precision to the Tevatron combined value. For comparison see Fig. 4 (right).

In order to reach the ultimate precision goal of less than $10 \mathrm{MeV}$ uncertainty, the missing 

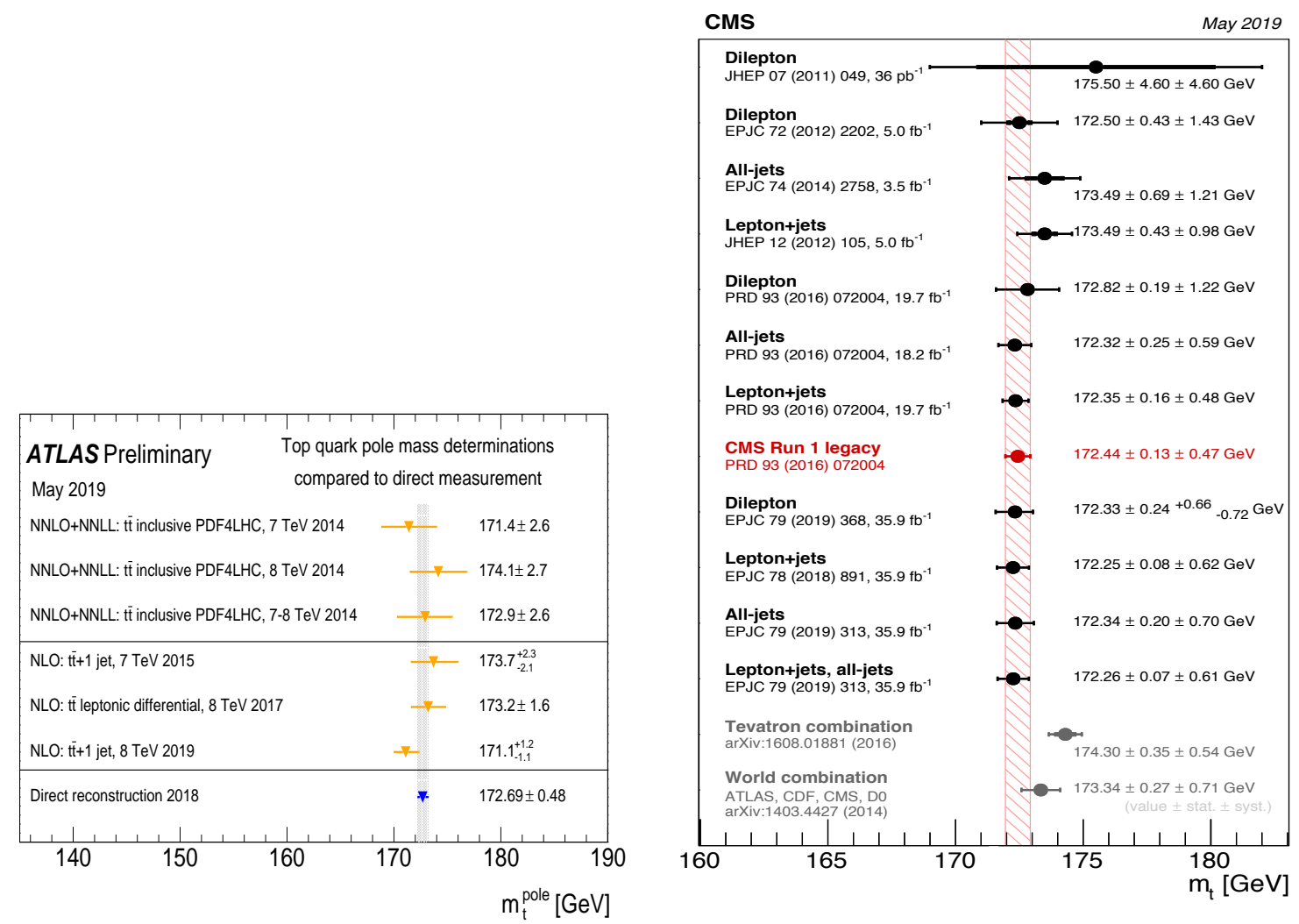

Figure 3: (left) Summary of the measurements of the top quark pole mass by ATLAS, compared to direct measurements [7]. (right) Summary of CMS top quark mass measurements. Tevatron and world combination (2014) results are also shown [8].
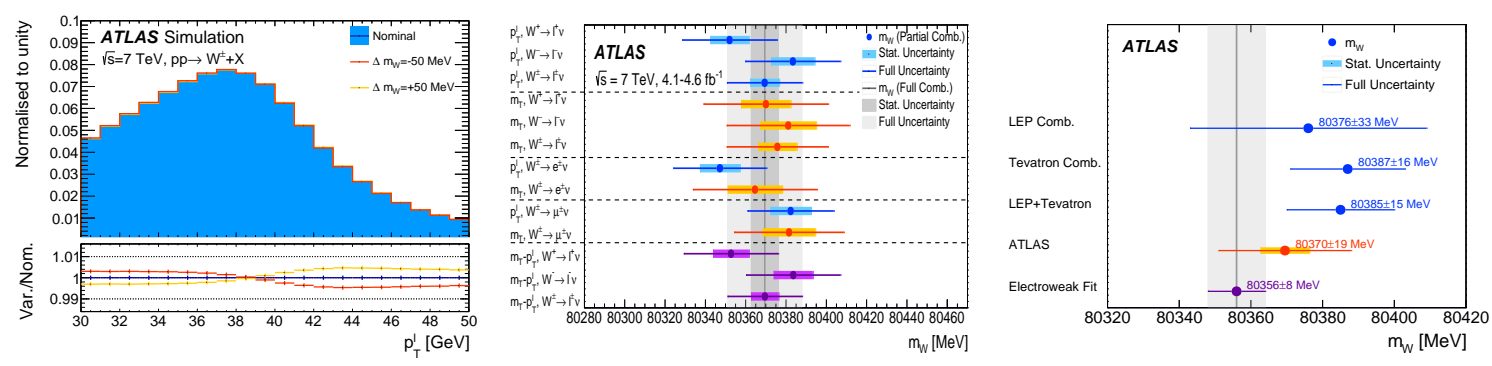

Figure 4: (left) Kinematic distribution of the lepton $p_{\mathrm{T}}$ in simulated events for the $W$ boson mass fitted value $m_{W}=80370 \mathrm{MeV}$ and for the values shifted by $\pm 50 \mathrm{MeV}$. (middle) Overview of the $W$ mass determinations from the lepton transverse momentum and the transverse mass distributions and from their combination, in the muon and electron decay channels and for $W^{+}$and $W^{-}$events. The statistical and total uncertainties are indicated. (right) The measured $W$ mass is compared to the SM prediction from the global electroweak fit by the Gfitter Collaboration from 2014 and to the combined results from the LEP and the Tevatron experiments. From Ref. [9]. 
transverse momentum reconstruction as well as the PDF information need to be improved. The upgraded detectors for HL-LHC would offer an opportunity as their finer segmentation would allow lower occupancy and the extended pseudorapidity coverage of the tracking system would contribute to an improved hadronic recoil calibration [11] as shown in Fig. 5 (right). Already with $300 \mathrm{pb}^{-1}$ data at low pile-up, the PDF uncertainty becomes dominant over the statistical one (see Fig. 5 (left)). HL-LHC will collect valuable PDF data, improving on today's precision by a factor of two, as illustrated on Fig. 5 (right). Even stronger constraints would be attainable from electron - proton collisions at LHeC, decreasing the PDF uncertainty to the 2-3\% level.
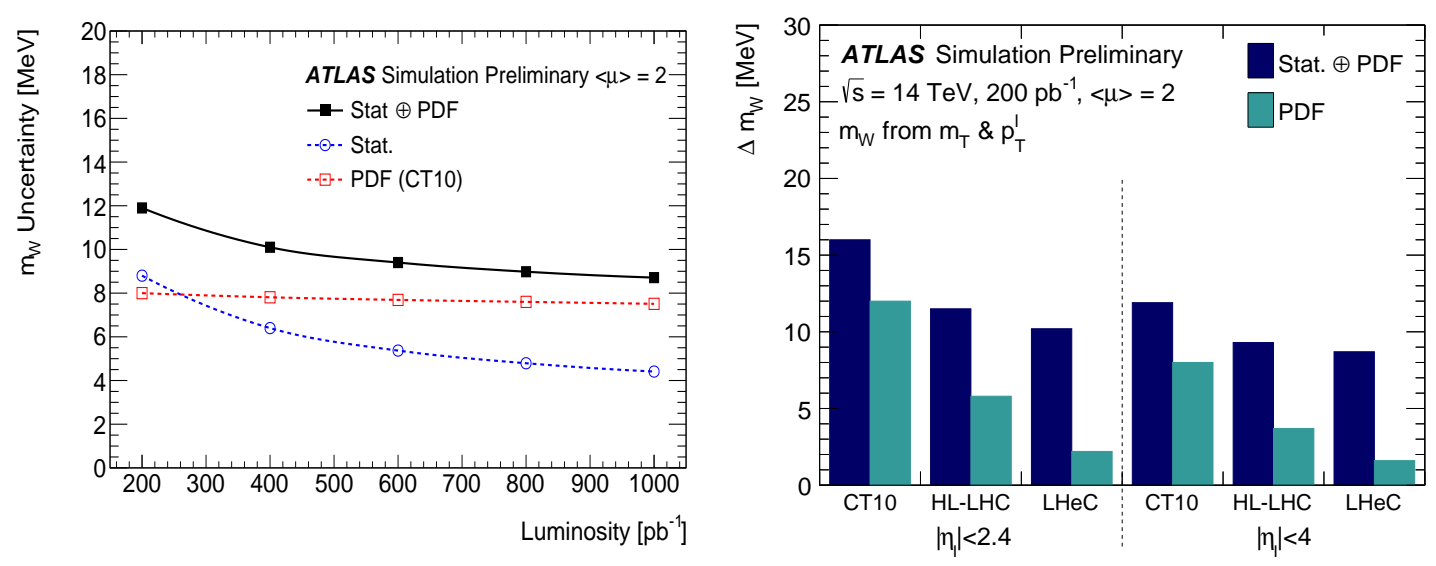

Figure 5: (left) Statistical and PDF uncertainty components as a function of integrated luminosity, for fully combined measurements at $\sqrt{s}=14 \mathrm{TeV}$, using the CT10 PDF set. (right) Measurement uncertainty for 200 $\mathrm{pb}^{-1}$ data collected within $\left|\eta_{\ell}\right|<2.4$ and $\left|\eta_{\ell}\right|<4$ for current CT10 and future PDF sets. From Ref. [11].

\section{4. $\mathbf{Z} \rightarrow \ell^{+} \ell^{-}$angular coefficients and forward-backward asymmetry}

The five-dimensional differential cross-section of $Z \rightarrow \ell^{+} \ell^{-}$can be factorised into the unpolarised cross-section $\left(\sigma^{U+L}\right)$ and the sum of nine harmonic polynomials describing the angular distribution (with $\theta$ denoting the polar and $\phi$ the azimuthal angle in the boson rest frame) with dimensionless angular coefficients $A_{0-7}\left(p_{\mathrm{T}}^{Z}, y^{Z}, m^{Z}\right)$ depending on the transverse momentum, rapidity and mass of the dilepton system:

$$
\begin{aligned}
\frac{\mathrm{d} \sigma}{\mathrm{d} p_{\mathrm{T}}^{Z} \mathrm{~d} y^{Z} \mathrm{~d} m^{Z} \mathrm{~d} \cos \theta \mathrm{d} \phi} & =\frac{3}{16 \pi} \frac{\mathrm{d} \sigma^{U+L}}{\mathrm{~d} p_{\mathrm{T}}^{Z} \mathrm{~d} y^{Z} \mathrm{~d} m^{Z}} \\
& \left\{\left(1+\cos ^{2} \theta\right)+\frac{1}{2} A_{0}\left(1-3 \cos ^{2} \theta\right)+A_{1} \sin 2 \theta \cos \phi\right. \\
& +\frac{1}{2} A_{2} \sin ^{2} \theta \cos 2 \phi+A_{3} \sin \theta \cos \phi+A_{4} \cos \theta \\
& \left.+A_{5} \sin ^{2} \theta \sin 2 \phi+A_{6} \sin 2 \theta \sin \phi+A_{7} \sin \theta \sin \phi\right\}
\end{aligned}
$$

The asymmetry in the total production cross-section of forward $(\cos \theta>0)$ and backward ( $\cos \theta<0)$ going leptons $A_{\mathrm{FB}}=\left(\sigma_{\mathrm{F}}-\sigma_{\mathrm{B}}\right) /\left(\sigma_{\mathrm{F}}+\sigma_{\mathrm{B}}\right)$ is related to the V-A structure of lepton 
couplings, and arises due to the $A_{4} \cos \theta^{*}$ term in Eq. 4.1, with $A_{\mathrm{FB}}=0.375 \cdot A_{4}$. As forwardbackward asymmetry depends on the dilepton mass, the weak mixing angle and the initial state quark flavour, its precise measurement around the $Z$ resonance peak can be used to derive $\sin ^{2} \theta_{\mathrm{W}}$ and the light quark weak couplings. Contributions to $A_{\mathrm{FB}}$ could also arise from new physics, for example the existence of new neutral gauge bosons, quark-lepton compositeness, supersymmetric partners of SM particles or extra spatial dimensions giving rise to new heavy states.

The measurements are performed in bins of the dilepton mass and rapidity in the Collins-Soper frame to reduce uncertainty from the transverse momentum of the incoming quarks. The extracted $A_{\mathrm{FB}}$ values are then fitted to derive the effective mixing angle defined by the ratio of the vector and axialvector couplings of the $Z$ boson to the fermions as $v_{f} / a_{f}=1-4\left|Q_{f}\right| \sin ^{2} \theta_{\text {eff }}^{f}$. It is related to the weak mixing angle $\sin ^{2} \theta_{\text {eff }}^{f}=k_{f} \sin ^{2} \theta_{\mathrm{W}}$, where $k_{f}$ is determined from higher order calculations. The method used by the CMS Collaboration is illustrated in Fig. 6 (top). The effective mixing angle is measured to be $\sin ^{2} \theta_{\text {eff }}^{\ell}=0.23101 \pm 0.00036$ (stat) \pm 0.00018 (syst) \pm 0.00016 (theo) \pm 0.00031 $(\mathrm{PDF})=0.23101 \pm 0.00053$, with the dominant uncertainty coming from the statistical error of the fit and the knowledge of the PDFs [12].

ATLAS extracts the $A_{4}$ angular coefficient by fitting the reconstructed $\cos \theta_{\mathrm{CS}}, \phi_{\mathrm{CS}}, m_{\ell \ell}$ and $y_{\ell \ell}$ distributions in Born-level $m_{\ell \ell}$ and $y_{\ell \ell}$ bins, after folding the theoretical $P_{i}\left(\cos \theta_{\mathrm{CS}}, \phi_{\mathrm{CS}}\right)$ angular distributions to detector level [13]. The measurement of $A_{4}$ in such a way in the full decay lepton phase space is dominated by statistical uncertainties. $\sin ^{2} \theta_{\text {eff }}^{\ell}$ then derived using the linear dependence shown in Fig. 6 (bottom left). The most precise measurement comes from the central - forward electron pair channel which gives access to leptons up to a pseudorapidy of $\left|\eta_{e}\right|=3.6$ and thus are the most sensitive for $A_{\mathrm{FB}}$ and $A_{4}$. The combined ATLAS result including also central - central electron pairs and muon pairs is $\sin ^{2} \theta_{\text {eff }}^{\ell}=0.23140 \pm 0.00021$ (stat.) \pm 0.00024 (PDF) \pm 0.00016 (syst.) $=0.23140 \pm 0.00036$. A summary of the results from various experiments is shown in Fig. 6 (bottom right).

\section{Drell-Yan differential cross-section measurements}

Drell-Yan lepton pair production provides a stringent test of higher-order electroweak and QCD predictions, it is sensitive to resummation techniques and constrains parton distribution functions that are a leading uncertainty in many LHC measurements. The precise measurement of lepton pairs from $Z$ (and $W$ ) decays are also essential for electroweak measurements, such as the $W$ mass and the weak mixing angle presented above. In fact, the knowledge of the $W$ boson $p_{\mathrm{T}}$ is a crucial ingredient for a $W$ mass measurement. The most precise $W p_{\mathrm{T}}$ distribution prediction is obtained by extrapolating from the measured $Z$ boson $p_{\mathrm{T}}$ distribution with $\mathrm{d} \sigma(W) / \mathrm{d} p_{\mathrm{T}}=\left[\left(\mathrm{d} \sigma(W) / \mathrm{d} p_{\mathrm{T}}\right) /\left(\mathrm{d} \sigma(Z) / \mathrm{d} p_{\mathrm{T}}\right)\right]_{\text {theory }} \times\left[\mathrm{d} \sigma(Z) / \mathrm{d} p_{\mathrm{T}}\right]_{\text {measured }}$.

The CMS measurements are performed with final state radiation corrected (so called dressed) leptons. The background contribution in the $Z / \gamma * \rightarrow \ell^{+} \ell^{-}$final state is low, as shown in Fig. 7 (left), getting contribution from tau pairs, diboson production and top quark production. A good agreement over the whole dilepton mass range is found with Standard Model predictions calculated by FEWZ at NNLO pQCD and NLO electroweak corrections with the NNPDF3.0 PDF set both on and off $Z$ resonance peak (Fig. 7 (middle)). At high masses the sizeable photon-induced contributions are also tested using FEWZ and LUXqed as shown in Fig. 7 (right). 

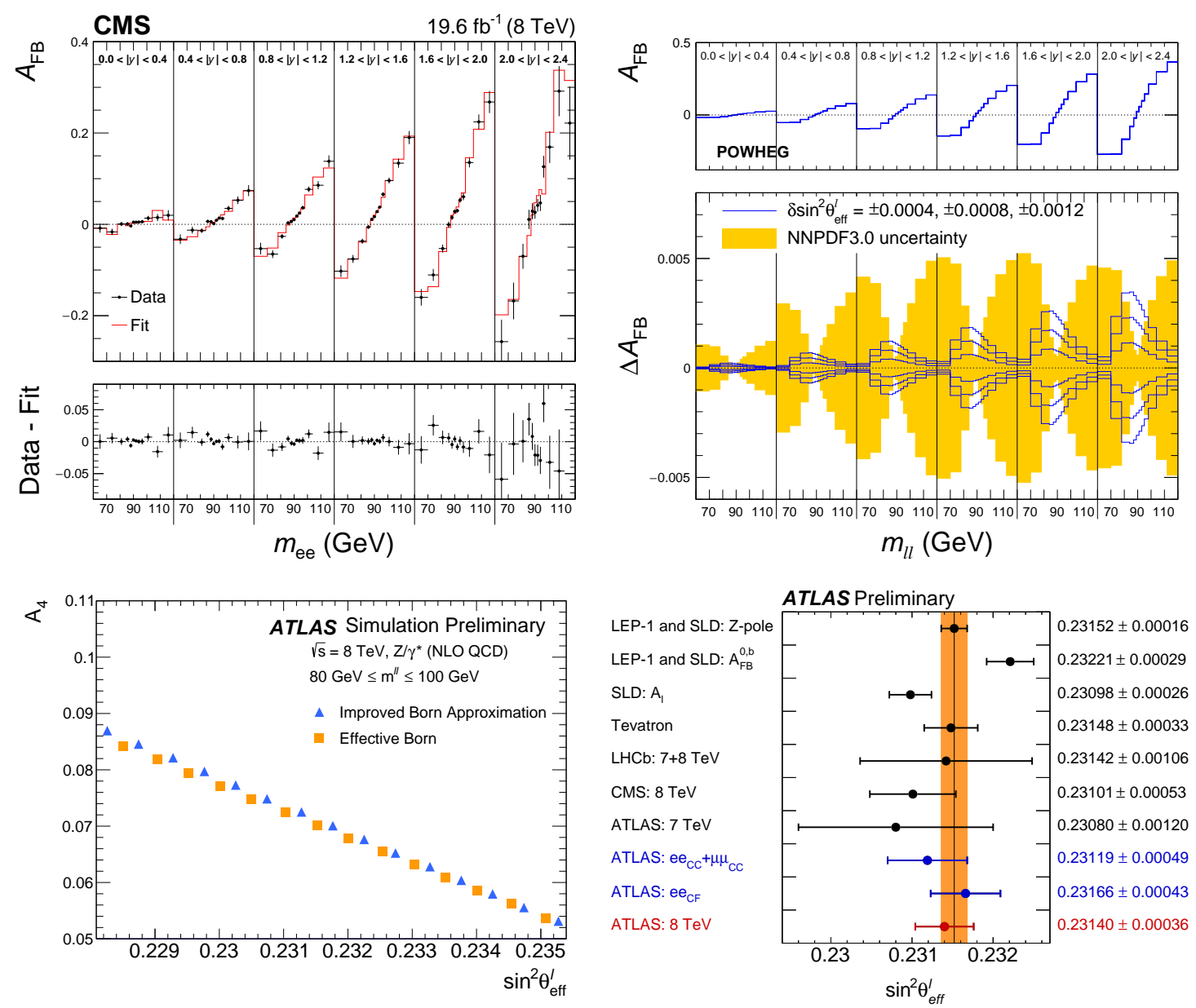

Figure 6: (top left) Comparison between data and best-fit $A_{\mathrm{FB}}$ distributions in the dielectron channel of the CMS analysis [12] as a function of dilepton mass, in six rapidity bins, using templates based on the central prediction of the NLO NNPDF3.0 PDF set. The error bars represent the statistical uncertainties in the data. (top right) $A_{\mathrm{FB}}$ dependence on the dilepton mass for $\sin ^{2} \theta_{\mathrm{eff}}^{\ell}=0.23120$ in POWHEG, from Ref. [12] . The solid lines in the bottom panel correspond to six changes at $\sin ^{2} \theta_{\text {eff }}^{\ell}$ around the central value. The shaded bands illustrate the standard deviation in NNPDF3.0 replicas. (bottom left) Predicted variation of $A_{4}$ as a function of $\sin ^{2} \theta_{\text {eff }}^{\ell}$ integrated over dilepton rapidity, transverse momenta and mass as predicted by the effective Born approximation with $\sin ^{2} \theta_{\mathrm{W}}=0.23152$ as well as by the improved Born approximation [13] . (bottom right) Comparison of the measurements of the effective leptonic weak mixing angle at various colliders, taken from Ref. [13].

Various differential cross-sections are studied by CMS [15] using the fiducial region of $\left|\eta_{\ell}\right|<$ $2.4, p_{\mathrm{T}}>25 \mathrm{GeV}$ and $\left|m_{\ell \ell}-91.2 \mathrm{GeV}\right|<15 \mathrm{GeV}$. The normalised cross-section as a function of the boson transverse momentum distribution is measured with an uncertainty below $0.5 \%$ for $p_{\ell \ell}<50 \mathrm{GeV}$ (as noted in the next section the normalisation has a dominant uncertainty from luminosity calibration). The results are corrected for detector effects and compared to various fixedorder, resummed and parton shower predictions in Figs. 8 and 9. The parton shower Monte Carlo generators use NLO QCD predictions for $Z$ (Powheg) and $Z+n$ jets $(n=0-1$ for Powheg+MiNLO and $n=0-2$ for MadGraph5_aMC@NLO, the latter using the FxFx scheme for matching). The 

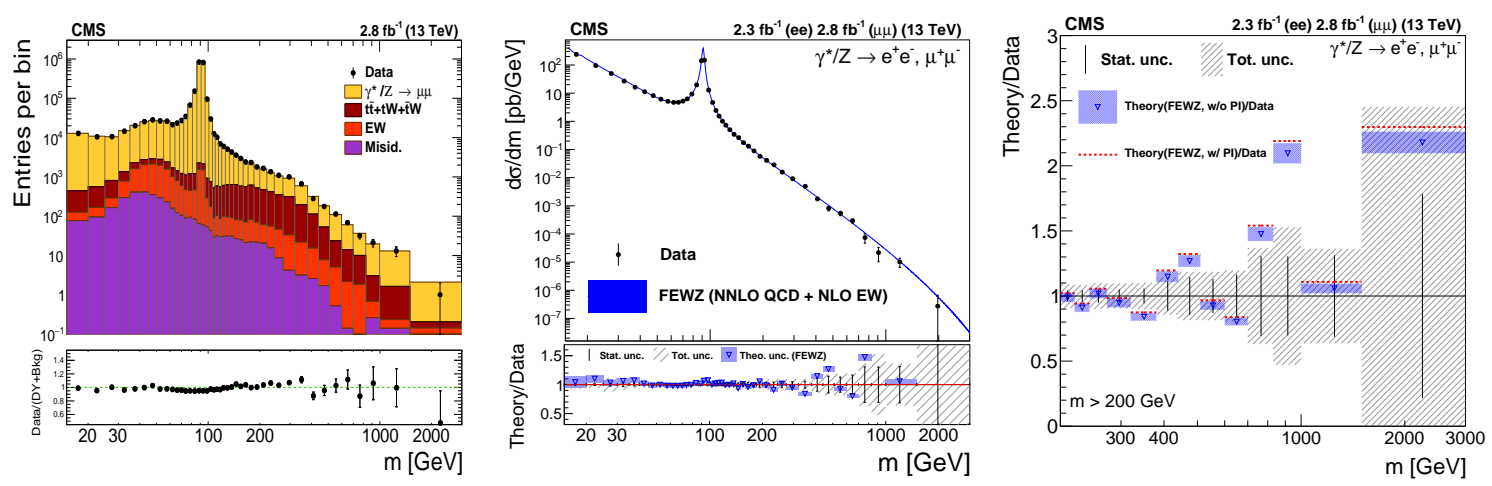

Figure 7: (left) The observed dimuon invariant mass spectrum. The electroweak background from the DY production of tau pairs, WW, WZ, and ZZ processes as well as the lepton misidentification contribution of $\mathrm{W}+\mathrm{jets}$ and QCD multijet processes are shown. The error bars on the data points represent the statistical uncertainty only. (middle) The differential DY cross-section measured by CMS and as predicted by the NNLO theoretical calculation of FEWZ in the full phase space. The coloured boxes represent the theoretical uncertainties.(right) The ratio of the NNLO theoretical prediction from FEWZ to data for the differential cross-sections in the $\mathrm{m}>200 \mathrm{GeV}$ mass range. The ratio with the photon-induced contribution (red dashed lines) is also shown. From Ref. [14].

fixed order FEWZ and ZjNNLO predictions are NNLO in perturbative QCD (pQCD) for $Z$ and $Z+$ jet production, respectively. Geneva and Resbos (not shown here) include leading order EW corrections and resummation at NNLL. Resbos can not describe the high- $p_{\mathrm{T}}$ region, while Geneva provides a reasonable description of the data. More details about the predictions and comparisons are in Ref. [15].
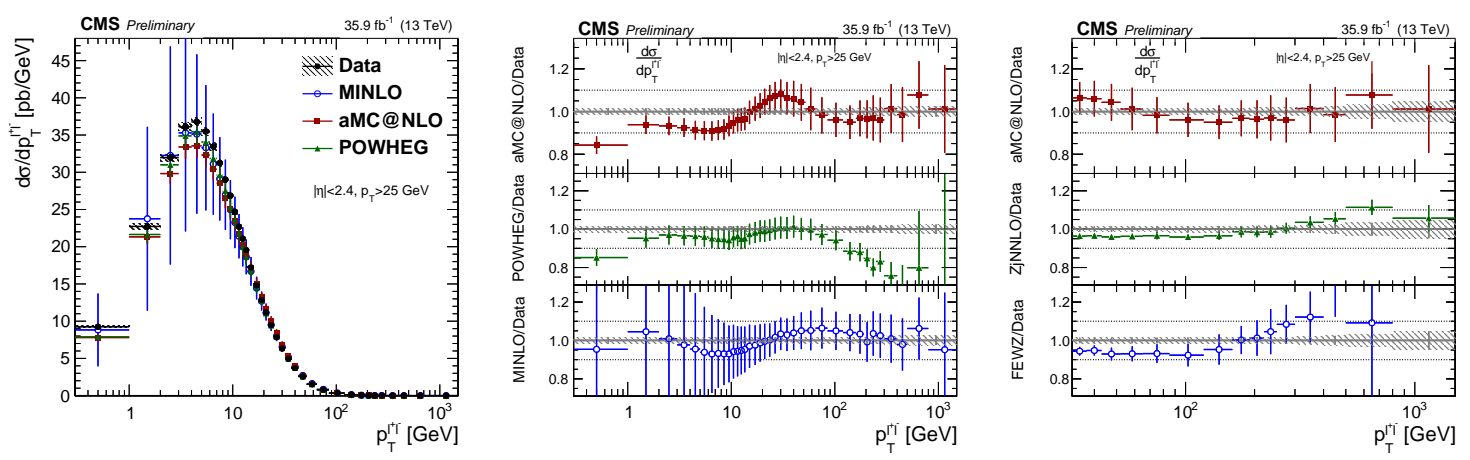

Figure 8: (left) The measured absolute cross-sections with total uncertainty bands in bins of dilepton transverse momentum, compared to various theoretical predictions. (middle - right) The ratio of cross-section predictions to the CMS data. The error bars around the predictions correspond to the combined statistical, PDF, and scale uncertainties. From Ref. [15].

\section{6. $W$ boson production and charge asymmtery}

As already mentioned, PDF uncertainties are one of the dominant contributions for precision measurements. The $W$ boson production charge asymmetry $\mathscr{A}_{\eta}=\left(\sigma_{\eta}^{+}-\sigma_{\eta}^{-}\right) /\left(\sigma_{\eta}^{+}+\sigma_{\eta}^{-}\right)$(with 

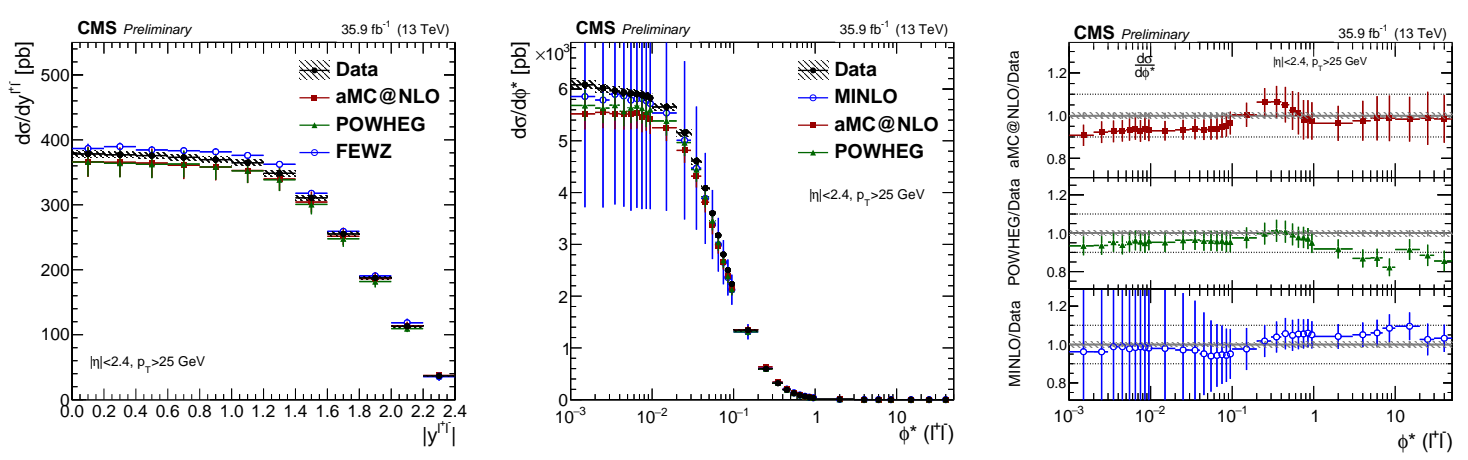

Figure 9: The measured absolute cross-sections with total uncertainty bands in bins of (left) dilepton rapidity and (middle) optimised angular variable $\phi^{*}$, compared to various theoretical predictions. (right) The ratio of cross-section predictions to the data in bins of $\phi^{*}$. The error bars around the predictions correspond to the combined statistical, PDF, and scale uncertainties. From Ref. [15].

$\sigma_{\eta}^{ \pm}$denoting the $W^{ \pm}$boson differential cross-section as a function of the pseudorapidity) allows the $u(x) / d(x)$ PDF ratio to be constrained in the Bjorken- $x$ region of $x=0.001-0.1$. The clean $W$ production signature (see Fig. 10 (left)) is thus ideal to test the various PDF sets. The cross-section measured by ATLAS, corresponding to a fiducial selection of $\left|\eta_{\mu}\right|<2.4, p_{\mathrm{T}, \mu}>25, p_{\mathrm{T}, v}>25$ $\mathrm{GeV}$ and $m_{\mathrm{T}}(\mu, v)>40 \mathrm{GeV}$ at Born level, is shown in Fig. 10 (middle). The asymmetry is then compared to DYNNLO with various PDF sets in Fig. 10 (right). The luminosity uncertainty (1.9\%) dominates, the sum of all other contributions being 1-1.5\% [16]. Reaching 1\% experimental precision would allow discrimination between the PDF sets.
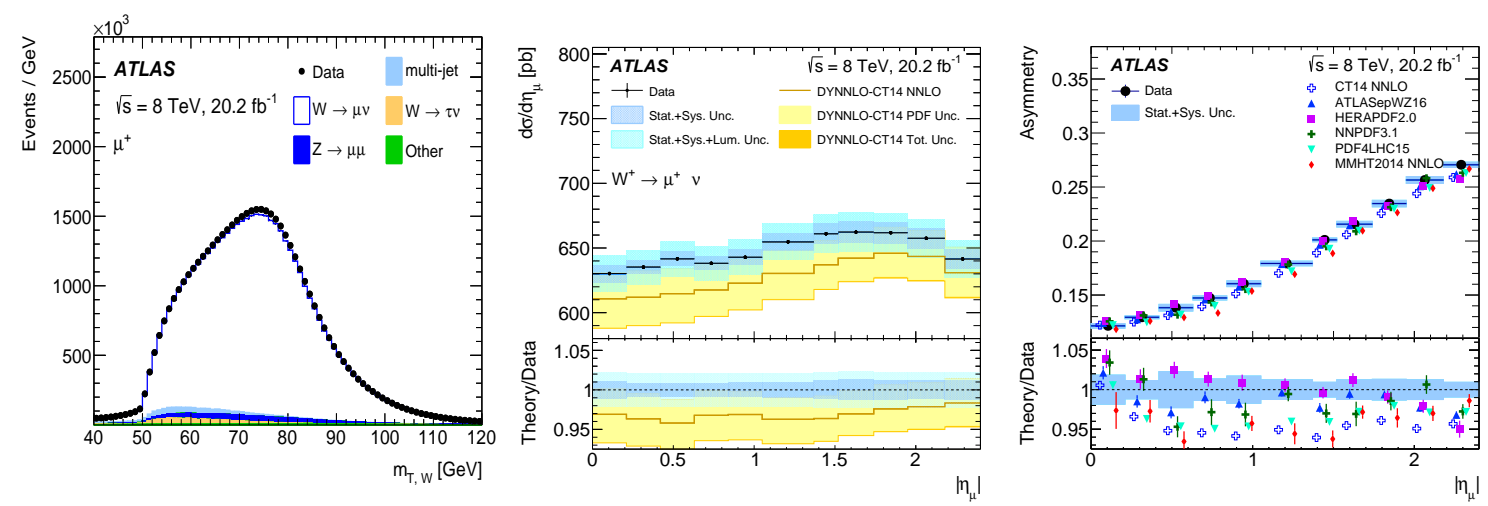

Figure 10: (left) The $W$ boson transverse mass (bottom) distribution of selected events with positive muons. The ATLAS data are compared to the theoretical prediction, showing also the background contributions. (middle) The $W^{+}$fiducial cross-section, differential in muon pseudorapidity, multiplied by the branching fraction for the decay into a muon and a neutrino. The data are compared with the predictions from DYNNLO using the CT14 NNLO PDF set. (right) The $W$ boson charge asymmetry as a function of absolute muon pseudorapidity. The data are compared to the central predictions from DYNNLO using a selection of PDFs. The statistical uncertainties of the DYNNLO predictions are indicated by error bars. The ratios of the data to the corresponding prediction are shown in the lower panel. From Ref. [16]. 


\section{Precision luminosity calibration}

For precision measurements with well controlled systematics, such as those using leptonic decays of the $W$ and $Z$ bosons and the top quark, a dominant systematic error arises from the integrated luminosity calibration, which typically has a $2-2.5 \%$ uncertainty per year, going down to $1.7-1.8 \%$ when all Run 2 data are combined. As illustrated in Fig. 11, this contribution would become subdominant in most cases if a $1 \%$ precision per year could be reached, this is thus the goal for the HL-LHC detector upgrades.
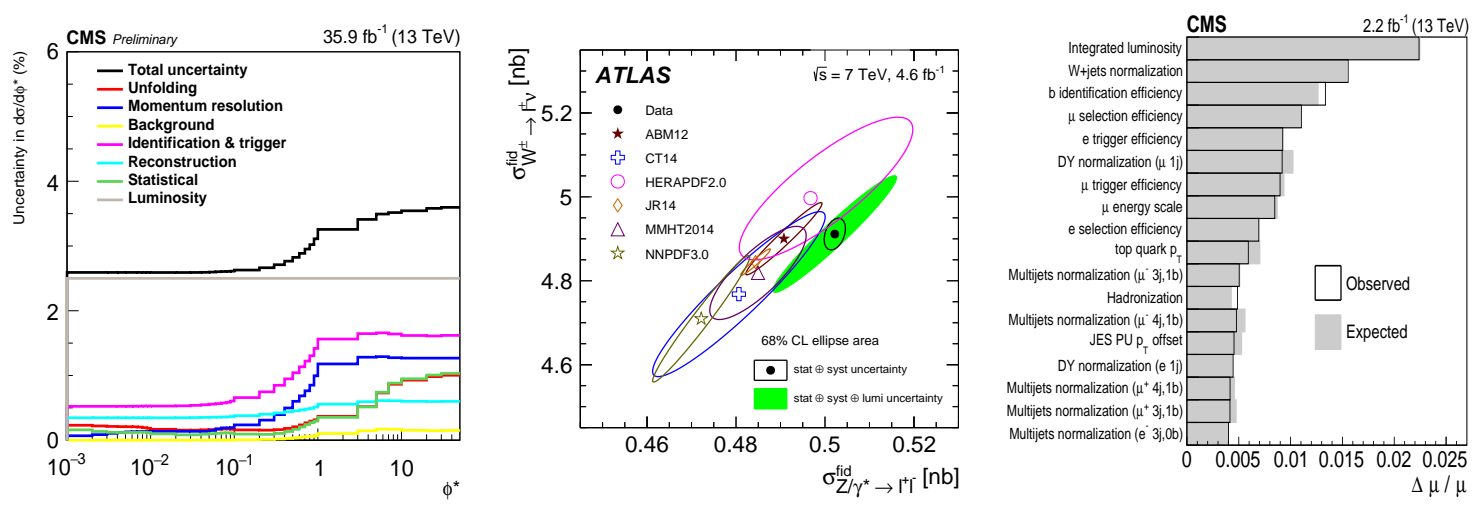

Figure 11: (left) The relative statistical and systematic uncertainties from various sources for the absolute $Z$ production cross-section measurements in bins of the angular variable $\phi^{*}$ in the dimuon final state in the CMS measurement [15]. The uncertainty in the trigger efficiency is included as part of the lepton identification uncertainty. (middle) Integrated fiducial cross-sections times leptonic branching ratios of $\sigma\left(W^{ \pm} \rightarrow \ell^{ \pm} v_{\ell}\right)$ vs. $\sigma\left(Z \rightarrow \ell^{+} \ell^{-}\right)$published by ATLAS in Ref. [17]. The data ellipses illustrate the $68 \%$ CL coverage for the total uncertainties (full green) and total excluding the luminosity uncertainty (open black). Theoretical predictions based on various PDF sets are shown with open symbols of different colours. The uncertainties of the theoretical calculations correspond to the PDF uncertainties only. (right) Estimated relative change in the measured signal strength $(\mu)$, coming from the various experimental and theoretical sources of uncertainties in the semi-leptonic top pair production analysis of CMS [18]. The open bars represent the values of the observed impact relative to the fitted signal strength. The values are compared to the expectations (shaded bars) by performing the fit using simulated events with $m_{t}=172.5 \mathrm{GeV}$. The various contributions are shown from the largest to the smallest observed impact.

\section{Summary}

ATLAS and CMS has an extensive program of Standard Model physics measurements covering five center-of-mass energies of 2.76, 5.02, 7, 8 and $13 \mathrm{TeV}$ that are compared to state-of-the-art higher order pQCD calculations manifesting excellent agreement as illustrated in Fig. 12 (top). While this paper concentrated on Drell-Yan type studies, there are many recent results on diboson production, summarised for example in Fig. 12 (bottom) [19]. In particular, vector boson fusion and scattering processes allow the measurement of anomalous electroweak triple and quartic gauge boson couplings due to New Physics and test the cornerstone of the model, the Brout-Englert-Higgs mechanism and its contribution to the regularisation of vector boson scattering amplitudes. 

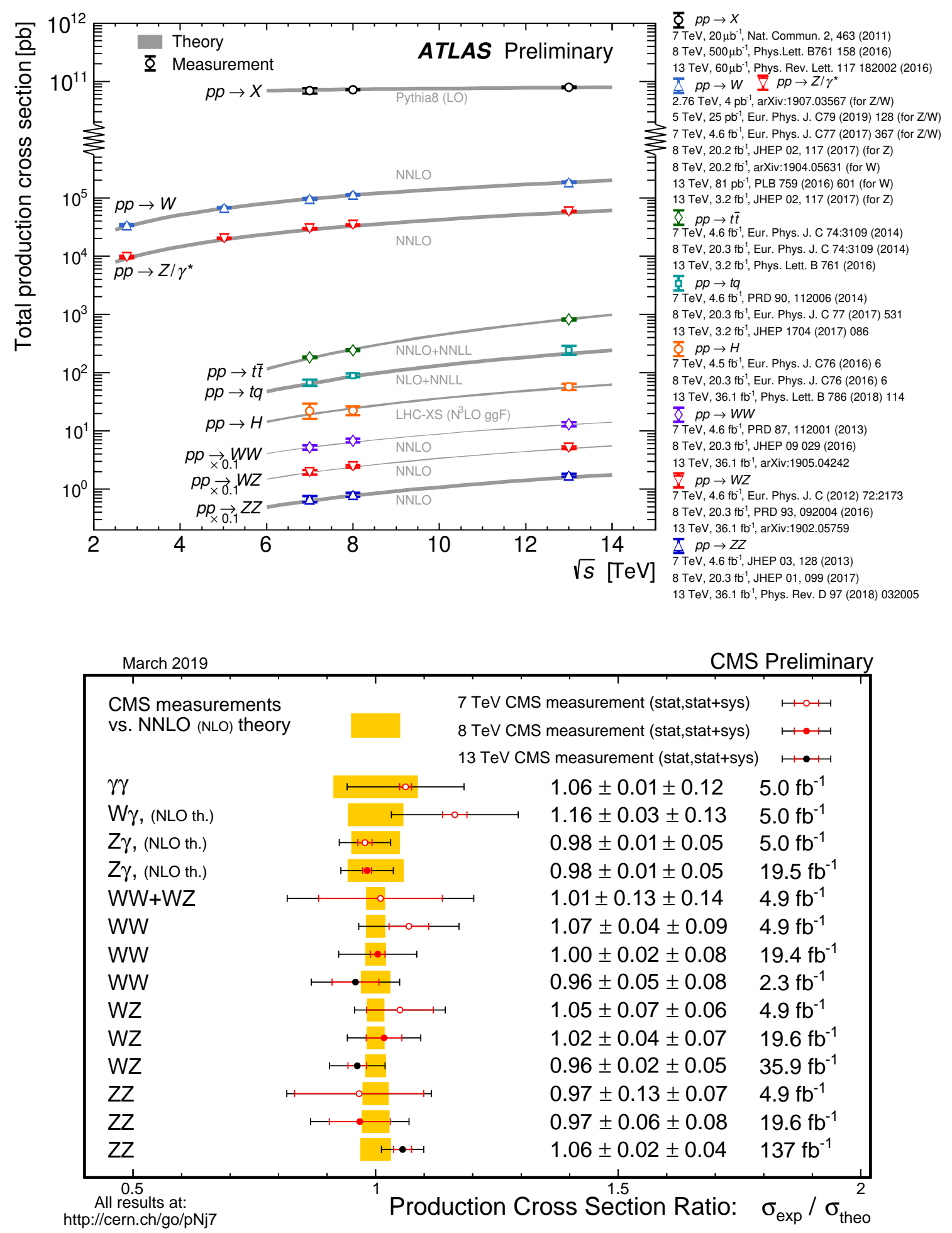

Figure 12: (top) Summary of total production cross-section measurements by ATLAS presented as a function of centre-of-mass energy from 2.76 to $13, \mathrm{TeV}$ for selected processes [6]. (bottom) Di-boson crosssection ratios to illustrate the comparison of CMS published and preliminary results to theoretical predictions updated to latest NNLO calculations where available [19]. 
The LHC became a precision machine reaching $\pm 19 \mathrm{MeV}$ uncertainty on the $W$ boson mass and \pm 0.0004 on $\sin ^{2} \theta_{\text {eff }}^{\ell}$, the individual experiments approaching the LEP/SLD and Tevatron combined precision. The contribution from the knowledge of parton distribution functions to the uncertainties is important and often dominant. The LHC results improved our understanding of the Standard Model and provide an essential test of its self-conistency, providing essential inputs to be the SM fits, such as the measurements of the masses of the $W$ boson, Higgs boson and top quark, the forward-backward lepton asymmetries and the effective weak mixing angle presented here.

\section{References}

[1] Andre Sopczak (on behalf of the ATLAS and CMS Collaborations), Precision measurements in the Higgs sector at ATLAS and CMS, to appear in these proceedings

[2] Particle Data Group, The Review of Particle Physics, Phys. Rev. D 98 (2018) 030001 and 2019 update at http://pdg.lbl.gov/pdg.html

[3] ATLAS Collaboration, The ATLAS Experiment at the CERN Large Hadron Collider, JINST 3 (2008) S08003

[4] CMS Collaboration, The CMS experiment at the CERN LHC, JINST 3 (2008) S08004

[5] Gfitter Collaboration, J. Haller, A. Hoecker, R. Kogler, K. Mönig, T. Peiffer, J. Stelzer, Update of the global electroweak fit and constraints on two-Higgs-doublet models, Eur. Phys. J. C78 (2018) 675, arXiv:1803.01853 latest results at http://project-gfitter.web.cern.ch/project-gfitter/Standard_Model/

[6] ATLAS Collaboration, Standard Model Summary Plots Spring 2019, ATL-PHYS-PUB-2019-010, latest results at https://twiki.cern.ch/twiki/bin/view/AtlasPublic/StandardModelPublicResults

[7] ATLAS Collaboration, Top Quark Mass Summary Plots (2019 May), ATL-PHYS-PUB-2019-020, latest results at https://twiki.cern.ch/twiki/bin/view/AtlasPublic/TopPublicResults

[8] CMS Collaboration, Summary of CMS top quark measurements (May 2019), latest results at https://twiki.cern.ch/twiki/bin/view/CMSPublic/PhysicsResultsTOPSummaryFigures

[9] ATLAS Collaboration, Measurement of the $W$-boson mass in pp collisions at $\sqrt{s}=7 \mathrm{TeV}$ with the ATLAS detector, Eur. Phys. J. C 78 (2018) 110, arXiv:1701.07240

[10] ATLAS Collaboration, Example ATLAS tunes of Pythia8, Pythia6 and Powheg to an observable sensitive to $Z$ boson transverse momentum, ATL-PHYS-PUB-2013-017

[11] ATLAS Collaboration, Prospects for the measurement of the $W$-boson mass at the HL- and HE-LHC, ATL-PHYS-PUB-2018-026

[12] CMS Collaboration, Measurement of the weak mixing angle using the forward-backward asymmetry of Drell-Yan events in pp collisions at 8TeV, Eur. Phys. J. C 78 (2018) 701, arXiv:1806.00863

[13] ATLAS Collaboration, Measurement of the effective leptonic weak mixing angle using electron and muon pairs from Z-boson decay in the ATLAS experiment at $\sqrt{s}=8 \mathrm{TeV}$, ATLAS-CONF-2018-037

[14] CMS Collaboration, Measurement of the differential Drell-Yan cross-section in proton-proton collisions at $\sqrt{s}=13 \mathrm{TeV}$, arXiv:1812.10529, submitted to JHEP

[15] CMS Collaboration, Measurements of differential $Z$ boson production cross-sections in pp collisions with CMS at $\sqrt{s}=13 \mathrm{TeV}$, CMS-PAS-SMP-17-010, now finalised in arXiv:1909.04133, submitted to JHEP 
[16] ATLAS Collaboration, Measurement of the cross-section and charge asymmetry of $W$ bosons produced in proton-proton collisions at $\sqrt{s}=8 \mathrm{TeV}$ with the ATLAS detector, Eur. Phys. J. C 79 (2019) 760, arXiv:1904.05631

[17] ATLAS Collaboration, Precision measurement and interpretation of inclusive $W^{+}, W^{-}$and $\mathrm{Z} / \gamma^{*}$ production cross-sections with the ATLAS detector, Eur. Phys. J. C 77 (2017) 367, arXiv:1612.03016

[18] CMS Collaboration, Measurement of the $t \bar{t}$ production cross-section using events with one lepton and at least one jet in pp collisions at $\sqrt{s}=13 \mathrm{TeV}$, JHEP 09 (2017) 051, arXiv:1701.06228

[19] CMS Collaboration, Di-boson cross-section ratio comparison to theory (2019 March), latest results at https://twiki.cern.ch/twiki/bin/view/CMSPublic/PhysicsResultsCombined 